\title{
Methodology and consistency of slant and vertical assessments for ionospheric electron content models
}

\author{
Manuel Hernández-Pajares · David \\ Roma-Dollase • Andrzej Krankowski • \\ Alberto García-Rigo • Raül Orús-Pérez
}

Received: date / Accepted: date

\begin{abstract}
A summary of the main concepts on Global Ionospheric Map(s) (hereinafter GIM(s)) of Vertical Total Electron Content (VTEC), with special emphasis on their assessment, is presented in this paper. It is based on the experience accumulated during almost two decades of collaborative work in the context of the International Global Navigation Satellite Systems (GNSS) Service (IGS) Ionosphere Working Group. A representative comparison of the two main assessments of ionospheric electron content models (VTEC-altimeter and difference of Slant TEC, based on independent Global Positioning System data GPS, dSTEC-GPS) is performed. It is based on 26 GPS receivers worldwide distributed and mostly placed on islands, from the last quarter of 2010 to the end of 2016. The consistency between dSTEC-GPS and VTEC-altimeter assessments for one of the most accurate IGS GIMs (the tomographic-kriging GIM 'UQRG' computed by UPC) is shown. Typical error RMS values of 2 TECU for VTEC-altimeter and 0.5 TECU for dSTEC-GPS assessments are found. And, as expected by following a simple random model, there is a significant correlation between both RMS and specially relative errors, mainly evident when large enough number of observations per pass is considered. The authors expect that this manuscript will be useful for new analysis contributor centers and in general for the scientific and technical community interested in simple and truly external ways of validating electron content models of the ionosphere.
\end{abstract}

M. Hernández-Pajares, A. García-Rigo

Universitat Politècnica de Catalunya (UPC-IonSAT), Barcelona, Spain

E-mail: manuel.hernandez@upc.edu

D. Roma-Dollase

Universitat de Barcelona, Dept. Electronics \& UPC-IonSAT, Barcelona, Spain

A. Krankowski

University of Warmia and Mazury in Olsztyn, Poland

R. Orús-Pérez

ESTEC, European Space Agency, Noordwijk, The Netherlands 
Keywords Satellite Navigation Systems · Ionosphere - Validation of ionospheric electron content models

\section{Introduction}

The multi-frequency Global Positioning System (GPS), and in general the Global Navigation Satellite Systems (GNSS), have become excellent ionospheric sounding systems in the last 20 years. This has been possible by exploiting the very well known predominant $(>99.9 \%$ ) ionospheric electron content dependence affecting to the transionospheric electromagnetic signals like those of GPS. This dependence is proportional to the integrated electron density and inversely proportional to the squared frequency (see for instance [Hernández-Pajares et al.2011], [Hernández-Pajares et al.2014]).

Dual-frequency GNSS measurements provide numerous simultaneous precise ionospheric delays in different directions and regions, and with an unprecedented temporal and spatial resolution (towards a delivery in Real-Time). This fact has been facilitated by the large number of transmitting satellites (typically larger than 20 GNSS satellites in view everywhere at any time) and many permanent networks of multi-frequency GNSS receivers. Indeed permanent networks providing data with rate of up to $1 \mathrm{~Hz}$ can be found at worldwide scale (like the International GNSS Service, IGS, network, see [Dow et al.2009]), at continental scales (like European EUREF, [Bruyninx2004], USA CORS, [Snay and Soler2008] or Australian CORS networks, [Janssen et al.2010]) and at regional scales (like CATNET network, [Talaya and Bosch1999], among many others). In this last category the very dense and wide area networks of Japan (GEONET, [Sagiya2004], with more than 1000 receivers) and South California (SCGIN, [Hudnut et al.2001], with several hundreds) are remarkable examples. This is due to their extremely high spatial sampling which allow, for instance, to directly view different kind of ionospheric waves. Examples of this last phenomenon, deserving increasing interest in the scientific community during the last years, are the common Medium Scale Travelling Ionospheric Disturbances (MSTIDs, see for instance a review in [Hernández-Pajares et al.2012]), or circular waves associated to strong earthquakes and/or corresponding tsunamis (see for instance [Galvan et al.2012]).

In such a context, GPS, which provides the majority of useful observations, will be the baseline for this work., The focus of this paper is on one of most popular ionospheric products in the Space Geodesy scientific and technological communities: the Global Ionospheric Maps. The GIMs are mostly computed from dual-frequency measurements gathered at global scale ([Schaer et al.1996],[Mannucci et al.1998], [Hernández-Pajares et al.1999]). Indeed, the GIMs are being systematically produced and openly provided by the IGS Ionosphere Working Group (IIWG) since June 1st, 1998 (see [Feltens2003], [Hernández-Pajares et al.2009]). They contain the worldwide distribution of the vertically integrated density (number of free electrons per volume unit) of 
the ionosphere (i.e. the so called Vertical Total Electron Content, VTEC), with a certain spatial and temporal resolution (usually $5^{\circ} \times 2.5^{\circ}$ and 15 minutes to 2 hours, respectively). The GIMS are typically estimated from a worldwide selected subset of hundreds of permanent GPS receivers, gathering thousands of dual-frequency GPS observations each time interval (such as 30 seconds), being accessible with a latency from less than 15 minutes (real-time experimental GIMs), 1 day (rapid GIMs) and up to 1-2 weeks (final GIMs).

Different estimation techniques have been developed by different ionospheric analysis centers, in particular for the slant-to-vertical mapping, with the general assumption of a common worldwide effective height or, alternatively, a tomographic description (see [Hernández-Pajares et al.1999]). Regarding to the very important aspect of interpolation, different techniques are used, like the spherical harmonic expansions, [SCHAER1999], and combined with generalized trigonometric series functions, [Li et al.2015], splines, [Mannucci et al.1998] and kriging, [Orús et al.2005] (see as well second column of Table 2). This last point is also relevant due to the large areas (such as the oceans, Siberia and most of the Souther Hemisphere) with very few available GPS receivers (see an overall study in [Hernández-Pajares et al.2009]).

The accuracy of the different techniques, in particular in the IIWG, has been continuously increasing in the last two decades, thanks to the open and daily-basis independent assessment, comparison, and combination with the corresponding weights in terms of a common IGS GIM. This common GIM is able to provide not only high accuracy, but especially very high reliability (availability and continuity) in all the different combination circumstances. In this regard it has been crucial to define fair, external and complementing assessment techniques. They have allowed the characterization of the strong and weak points of every GIM at different distances of the permanent receivers (see [Hernández-Pajares2004]). Summary and, by the first time, direct comparison of those is the main target of this paper. In this way we expect to facilitate both, the incorporation of new analysis centers to IIWG contributing with new GIMs to hopefully further improve the combined IGS GIM, and in general the provision of a summary of simple and useful ways of performing truly external validations of electron content models.

\section{GIM concepts}

Let us first summarize some main ideas and suggestions about GIMs, which have been very useful to increase the accuracy of GIMs during the almost 20 years of collaborative work within the IIWG:

1. The big challenge since the beginning in IIWG was that the GIMs should have no lack of availability or "holes". Then the ionospheric interpolation techniques become crucial, especially in the large regions with sparse coverage of permanent GPS receivers. In this context the usage of, for example, a solar-magnetic reference frame (where the electron distribution is most stationary), or accurate interpolation techniques like Kriging (see 
for instance the above mentioned reference [Orús et al.2005]), among other accurate techniques, become important (see Figure 1).

2. The reliability and accuracy of the combined IGS GIM is mostly based on the fair assessment of the consistency and accuracy of the individual GIMs, provided by different IIWG centers (four during the last 12 years: CODE, ESA-ESOC, JPL and UPC, see [Hernández-Pajares et al.2009]).

3. The GIM accuracy shall be assessed from independent electron content data, free from unknown biases, and ideally not taking part in the GIM computation.

4. Moreover the assessment method should be directly related to the physical quantity (the electron content) provided within the GIMs (for instance this point and the previous one are not fulfilled in [Rovira-Garcia et al.2015]).

In this context we will summarize in the next section two successful GIMindependent assessment procedures, which complement each other well:

1. Taking as reference direct VTEC measurements from dual-frequency altimeters (available over the oceans) on one hand, and

2. Taking reference measurements of variations of slant ionospheric delay from permanent GPS receivers which have not been involved in the GIM computation (typically within or closer to continents, large islands and existing GPS networks), on the other hand.

It is important to note that the GIMs computation is then quite different from the computation of Regional or either continental scale Ionospheric Models (hereinafter RIMs), based only on GPS data gathered in regions with a good coverage of GPS receivers (such as Europe or USA). In these scenarios the accuracy of the ionospheric delay provided by RIMs can be higher, due to the higher density of measurements (see for instance [Hernández-Pajares et al.2000], [Colombo et al.2002], [Juan et al.2012]).

\section{GIM assessment methods}

Two methods have been successfully used to assess GIMs for almost two decades and they are still in use today:

1. The comparison with direct VTEC measurements provided by dual-frequency altimeters (taken in slightly different frequency bands than GNSS, and with a typical measurement error standard deviation after smoothing of about 1 TECU, see Figure 2). This reference VTEC is obtained up to the height of the altimeters (typically $+1300 \mathrm{~km}$ ) which includes the full ionosphere and the most contribution of the plasmaspheric electron content.

The noise of the altimeter measurements can be significantly reduced by a sliding window (of $16 \mathrm{sec}$. in the example shown in top plot of Figure 3, see Figure 2 for overall distribution of Standard Deviation after smoothing). Moreover, comparing the altimeter VTEC vs rapid UPC VTEC, the missing altimeter-topside electron content is just a small part of plasmaspheric 
Day 317,2012

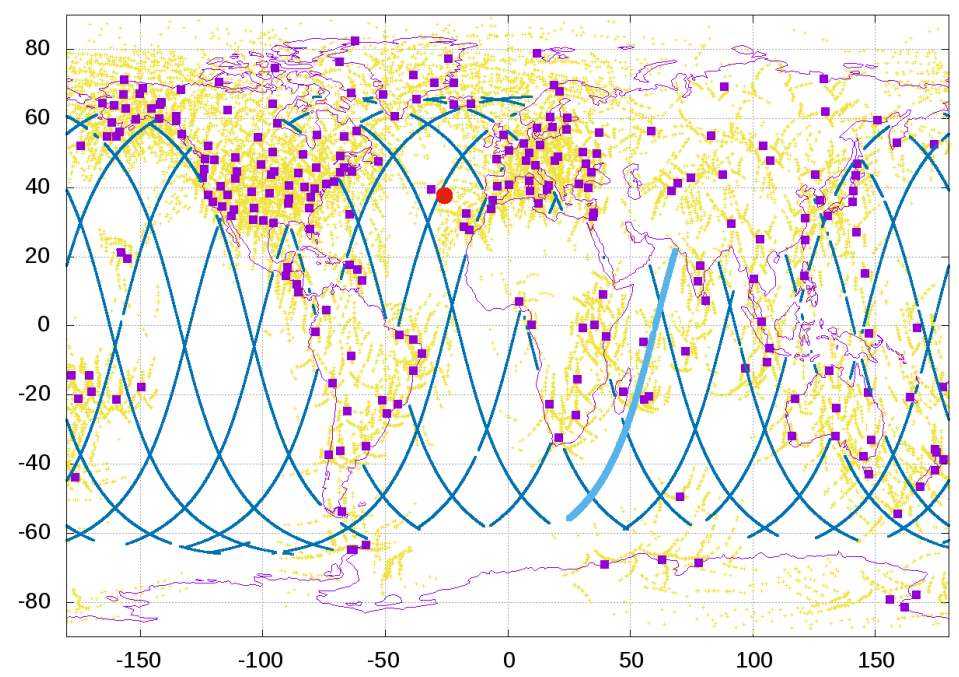

VTEC[UQRG] / TECU for Day 112 (Nov 12th), 2012, 16h45m GPStime

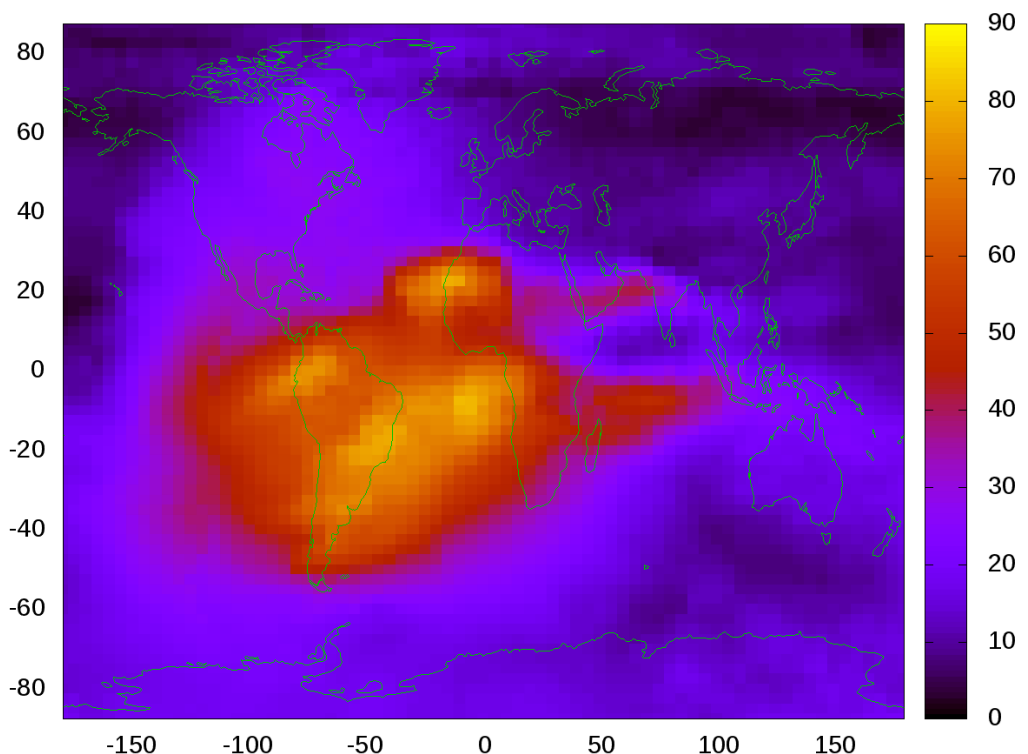

Fig. 1 Upper plot: It can be seen for day 317 (Nov. 12th), 2012, the map representing the GPS receivers contributing to the UPC VTEC GIM "UQRG" (computed with a tomographic-kriging technique following [Hernández-Pajares et al.1999] and [Orús et al.2005], magenta squares), the ionospheric pierce points (IPPs) of the corresponding observations from $16 \mathrm{~h} 15 \mathrm{~m}$ to $17 \mathrm{~h} 15 \mathrm{~m}$ (yellow points), the JASON2 altimeter IPPs (blue lines, emphasizing in thick-blue one typical JASON2 pass to be detailed in next Figure) and an example of external permanent receiver (PDEL, red circle) suitable for assessing dSTEC. Bottom plot: For the same day 317 (Nov. 12th), 2012, the VTEC global distribution is represented in TECU, obtained from the UPC GIM UQRG, corresponding to the selected JASON2 pass (16h45m GPS time). 
Sel repres_days_2010-2016

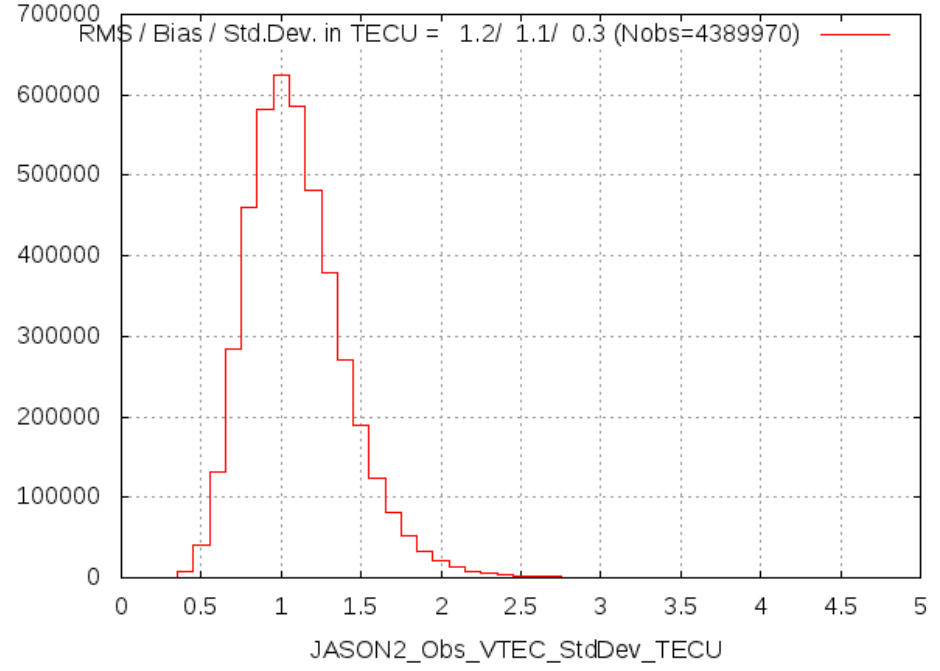

Fig. 2 Histogram representing the distribution of the JASON2 VTEC Standard Deviation, associated to the sliding window smoothing, for the representative datasets analyzed in this study, since 2010 to 2016 (more than 4,000,000 JASON2 observations analyzed).

component (above $+1300 \mathrm{~km}$ and typically up to few TECUs only). Finally, the well know altimeter bias excess (see [Azpilicueta and Brunini2009]), presents a value of few TECUs only in such a way that it still allows a very clear assessment and comparison of the errors of the different ionospheric models (considering for instance daily statistics), typically much larger and systematic. This is the reason why the dual-frequency altimeter measurements provide an excellent and independent source for assessing GNSSbased VTEC models in difficult conditions, overseas and typically far from receivers (see for example [Hernández-Pajares2004], [Orús et al.2005], [Hernández-Pajares et al.2009] and [Hernández-Pajares et al.2016]) This is illustrated in the example given at the top plot of Figure 3, which corresponds to a typical JASON2 pass, in this case crossing the two equatorial anomaly peaks (see track in thick-blue at the top plot of Figure 1). It is evident the good agreement of the GIM VTEC with the altimeter VTEC for the most part of the track (excepting for one of the equatorial anomaly peaks -around latitude $+18^{\circ}$-, a most difficult modelling region).

2. The comparison with directly observed STEC variations along a phasecontinuous transmitter-receiver arc, provided from GPS receivers with accuracies $\leq 0.1$ TECU $^{1}$, is another well-behaving and useful electron con-

1 This typical maximum error can be deduced from the $2 \mathrm{~mm}$ of nominal carrier phase measurement noise (see page 4.15 in [Wells et al.1987]), the definition of geometry-free combination of both carrier phases L1-L2, and the two STEC measurements involved in dSTEC, after taking into account that the carrier phase multipath is typically very small. 

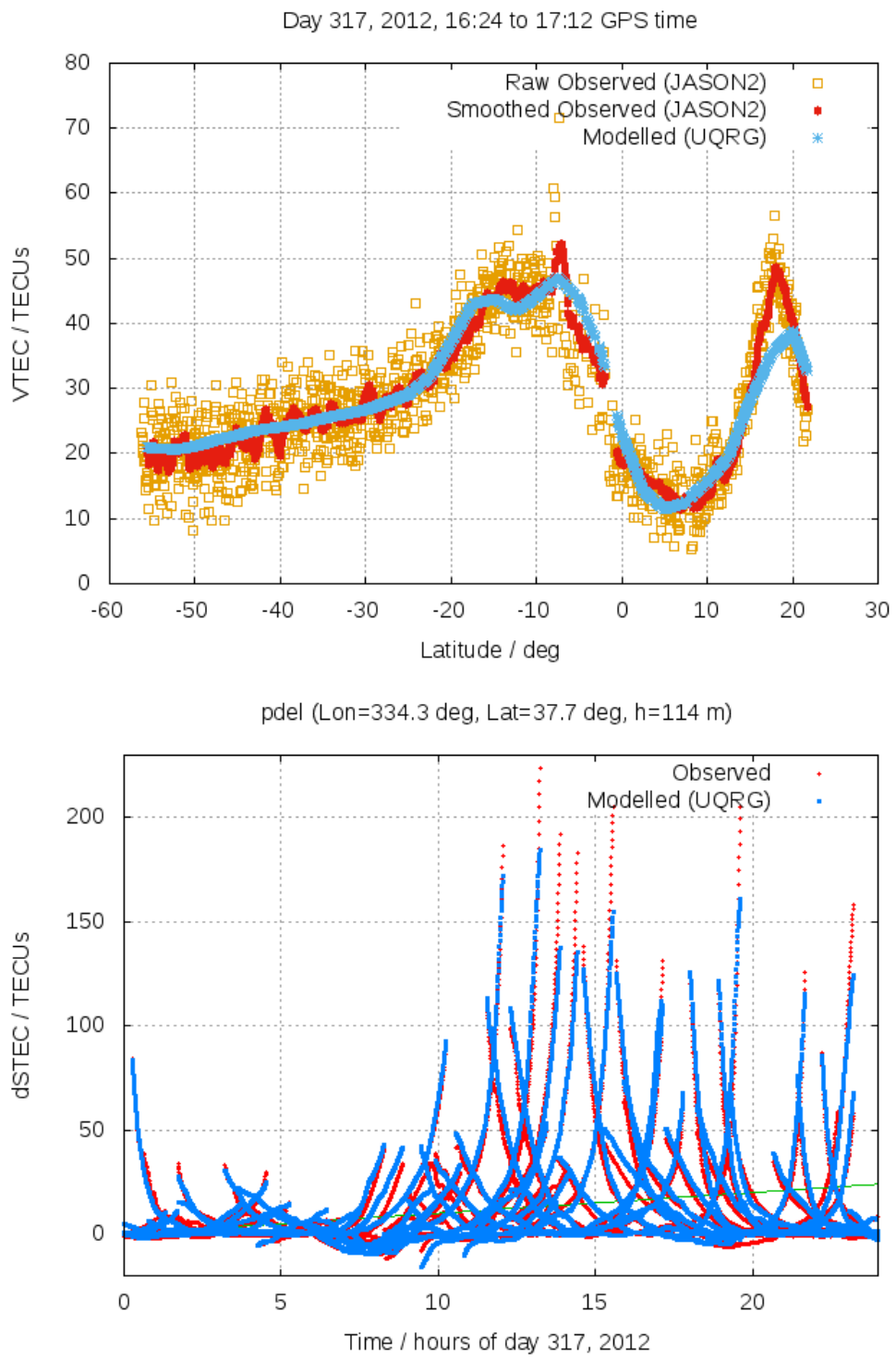

Fig. 3 Top plot: Example of altimeter (JASON-2) VTEC (both raw and smoothed observations) vs. GIM ("UQRG" by UPC) VTEC comparison (same day as previous figure, day 317 (Nov. 12th), 2012, GPS time 16h24m-17h12m GPS time). Bottom plot: dSTEC Observed (red) and Modelled with UQRG GIM (blue) corresponding to the GIM-external GPS receiver PDEL, located in the top map of Figure 1, during the same day 317 (Nov. 12th), 2012. 
Table 1 Comparison of main favourable factors (pros) and the unfavourable factors (cons) for VTEC altimeter and dSTEC GPS data.

\begin{tabular}{|c||c||c|}
\hline Technique & Favourable factors (PROS) & Unfavourable factors (CONS) \\
\hline VTEC Altimeter & Indep. VTEC assessment & Only over oceans \\
\hline dSTEC GPS & Indep. STEC assessment & Mainly over land \\
\hline
\end{tabular}

tent modelling assessment test. It has been used since 1998 for ranking, weighting and combining the GIMs of IGS centers in terms of a common IGS GIM, by considering the error in providing the STEC difference in the phase-continuous transmitter-receiver arc among two observations with a similar elevation at the opposite place of the arc (see "selfconsistency test" in [Orús et al.2005]). In our case we use a slightly different method (hereinafter dSTEC), which is defined just changing the reference ray in the phase-continuous transmitter-receiver arc (the one with the highest elevation instead of the observation with a similar elevation, see [Orús2005] -where it was introduced as "Maximum Elevation test"-, see [Feltens et al.2011] and below for more details).

The dSTEC test complements the altimeter VTEC test (see Table 1): First, dSTEC assesses in slant directions, instead of vertically, like the VTEC-altimeter test. And secondly, dSTEC is available close to GPS receivers, in spite of being far from them, like typically the altimeter VTEC measurements over the oceans. But, as was commented above, it is very important in the dSTEC assessment to use GPS receivers as reference GPS which have not been used by any of the GIMs to be compared, to avoid unclear assessments. Examples of such comparison can be seen in [Feltens et al.2011] and [Hernández-Pajares et al.2016], where 4 and 9 different ionospheric models are compared, respectively.

Indeed, dSTEC (hereinafter $\Delta S$ in the equations), is defined as the difference of the given STEC and the STEC at the highest elevation, for each given pair of transmitter and receiver, and for a common arc of measurements. This definition tries to offer a proxy for assessing the behaviour of the modelled STEC, in two different directions and times, because typically the highest elevation ray has lower errors, in particular due to the much less relevance of the mapping function (however the self-consistency test assess the observed accurate difference of STEC for two rays separated in time and space but with the same elevation, as it was indicated above).

The good news is that $\Delta S$ can be directly obtained with almost no-effort from the dual-frequency carrier phase combination $L_{I}=L_{1}-L_{2}$ of the raw dual-frequency GPS carrier phases $\left(L_{1}\right.$ and $\left.L_{2}\right)$ when no cycle slips happen (typically up to few hours in permanent GPS receivers), following Equation 1, in which the small term of carrier phase windup of $L_{I}$ (less than 0.25 TECUs for ground permanent GPS receivers) is considered corrected (see an example of measured dSTEC values at bottom plot in Figure 3): 


$$
\Delta S_{o}=S_{o}(t)-S_{o}\left(t_{\mathrm{Emax}}\right)=\frac{1}{\alpha}\left(L_{I}(t)-L_{I}\left(t_{E_{\max }}\right)\right)
$$

where $\alpha=\frac{q^{2}}{3 \pi^{2} m_{\mathrm{e}} \epsilon_{0}} \simeq 40.3$ in S.I. units, being $q$ and $m_{\mathrm{e}}$ the charge and mass of the electron, respectively, and $\epsilon_{0}$ the dielectric constant in the vacuum (see for instance [Hernández-Pajares et al.2010]).

Then $\Delta S$ can be used to compare the performance of approximating the observed value $\Delta S_{o}$ in terms of the value provided by each given ionospheric model, $\Delta S_{m}$. In this regard we will focus on $R M S\left[\Delta S_{o}-\Delta S_{m}\right]$, comprising a wide interval of elevations in each given arc of data, but clearly below $E_{\max }$. Indeed the observed dSTEC is a direct and very accurate measurement of the difference of STEC directly derived from the dual-frequency carrier phases, involving different geometries (elevation angles and regions of the ionosphere) and different times. Then this is a convenient test, when external receivers are considered, for any ionospheric model, regarding the accuracy of its mapping function, the time-dependence and the electron content determination itself.

In the next section we present a dedicated study to characterize how compatible both tests are, by processing a representative dataset in a worldwide set of 26 GPS receivers mostly placed on islands, i.e. collocated with VTEC altimeter data.

\section{Comparison of VTEC-altimeter and dSTEC-GPS assessments}

Our purpose is to check the consistency between the assessments of VTEC GIMs by means of VTEC-altimeter and dSTEC-GPS observations, by comparing them when the corresponding measurements are collocated. And in this regard some important aspects should be considered in this new study:

1. In order to have the clearest picture of the assessment comparison we should select one of the best available VTEC GIMs, in order to prevent an important GIM error jeopardizing it. We have adopted the rapid UPC GIM (latency of one day), with a resolution of 15 minutes, $5^{\circ}$ and $2.5^{\circ}$ in time, longitude and latitude respectively. Such GIM (with IGS identification "UQRG") is computed with the UPC TOMION software, by means of a tomographic and kriging combined technique (see [Orús et al.2005], [Hernández-Pajares et al.1999]) and it is behaving in particular better than the official UPC GIM: see Table 2 where a recent combined assessment of GIMs has been separately performed with both VTEC-altimeter and dSTEC-GPS data (more details can be found in [Hernández-Pajares et al.2016]).

2. The target of comparing VTEC-altimeter with dSTEC-GPS data brings us to consider GPS receivers on islands: in particular 26 worldwide distributed GPS receivers, mostly over islands, have been selected during 102 days evenly distributed from last quarter of 2010 to end of 2016 (see Figure 4). This period, constrained by the availability of the above introduced UQRG 
Table 2 Recent GIMs assessment vs VTEC-altimeter. It is based on a common set of 21 days with available JASON2 observations, on the one hand, and on dSTEC-GPS, providing the daily RMS over +50 independent GPS receivers. The relative error of ionospheric model for VTEC (versus the JASON2 VTEC) and for dSTEC (vs the GPS dSTEC) are included in columns 3 and 4, respectively (extracted from [Hernández-Pajares et al.2016], where more details can be found).

\begin{tabular}{|c|c|c|c|c|}
\hline $\begin{array}{l}\text { GIM } \\
\text { Id. }\end{array}$ & $\begin{array}{l}\text { Method \& } \\
\text { Reference }\end{array}$ & $\begin{array}{c}\text { VTEC[alt.] - VTEC[GIM] } \\
\text { Rel. Error } / \% \\
\text { [21 common days in } \\
2015,117-2016,007]\end{array}$ & $\begin{array}{c}\text { dSTEC }[\text { GPS }] \text { - dSTEC }[\text { GIM }] \\
\text { Rel. Error } / \% \\
\text { [year } 2015 \text {, days } \\
082,146,280 \& 330]\end{array}$ & $\begin{array}{c}\# \\
\text { Rec* } \\
\text { Days }\end{array}$ \\
\hline IGSG & $\begin{array}{c}\text { Weighted } \\
\text { mean [Hernández-Pajares et al.2009] }\end{array}$ & 21.1 & 28.9 & 238 \\
\hline CODG & $\begin{array}{c}\text { Sph. Harm. } \\
(\mathrm{SH})[\mathrm{SCHAER} 1999]\end{array}$ & 21.8 & 27.8 & 238 \\
\hline ESAG & SH [Feltens2007] & 25.5 & 33.0 & 238 \\
\hline JPLG & $\begin{array}{c}\text { Three-shell } \\
\text { model [Mannucci et al.1998] }\end{array}$ & 21.9 & 31.0 & 180 \\
\hline UPCG & $\begin{array}{c}\text { Two-layer } \\
\text { voxels [Hernández-Pajares et al.1999] }\end{array}$ & 19.1 & 26.9 & 238 \\
\hline CASG & $\begin{array}{c}\text { SH \& } \\
\text { GTS [Li et al.2015] }\end{array}$ & 21.1 & 28.0 & 178 \\
\hline EMRG & SH [Ghoddousi-Fard et al.2011] & 26.5 & 33.6 & 178 \\
\hline WHUB & $\begin{array}{c}\text { SH \& } \\
\text { ICLS [Zhang et al.2013] }\end{array}$ & 25.0 & 30.7 & 60 \\
\hline UQRG & $\begin{array}{c}\text { Tomogr. \& } \\
\text { Kriging [Orús et al.2005] }\end{array}$ & 16.3 & 20.5 & 233 \\
\hline
\end{tabular}

GIMS (likely the most accurate GIMs presently available, see Table 2), covers the most part of variability of the present Solar Cycle (see Figure 5).

3. As the result of a compromise between spatio-temporal colocation and VTEC-altimeter and dSTEC-GPS data availability, the following main requirements have been considered to select the altimeter passes (JASON-2 in our experiment): The maximum difference of longitudes, latitudes and times, among the ionospheric pierce points of the altimeter and slant GPS measurements, should be smaller than $12^{\circ}, 10^{\circ}$ (coinciding with latitudinal tick marks interval in top plot of Figure 3) and 900 seconds, respectively. This means 6407 passes with at least 25 VTEC-JASON2 and dSTEC-GPS collocated observations (see distribution in time and latitude of the overall passes in Figure 6).

The assessment comparison is firstly summarized in Figure 7 with the plot of dSTEC-GPS assessment error and VTEC-altimeter assessment error of UQRG GIM versus time and latitude (left- and right-hand plot respectively). Both VTEC and dSTEC errors evolves in a compatible way along the analyzed period of more than 6 years, showing up higher values in agreement with the 


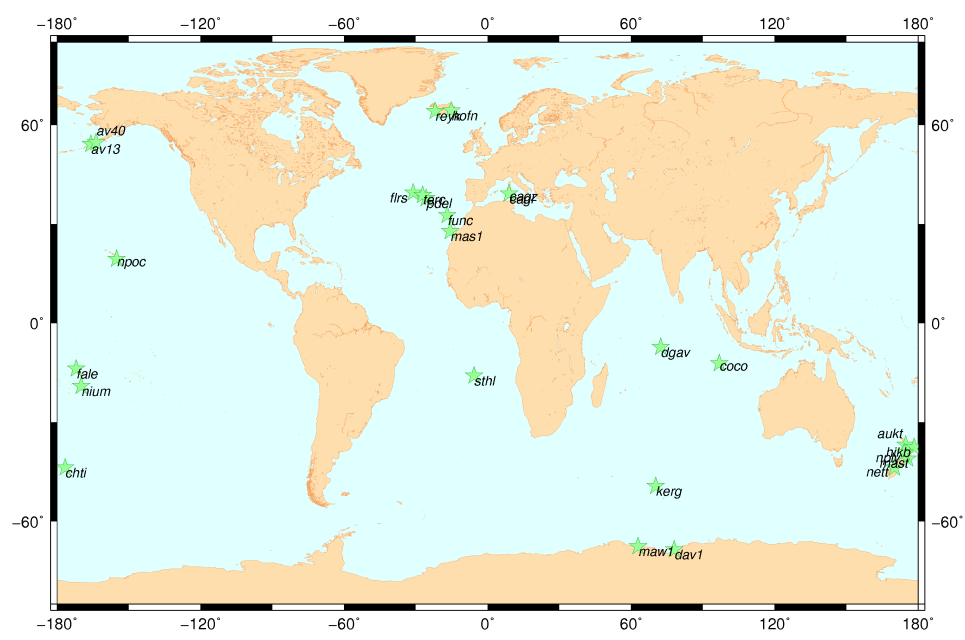

Fig. 4 Selected set of 26 GPS receivers located on islands and coast lines.

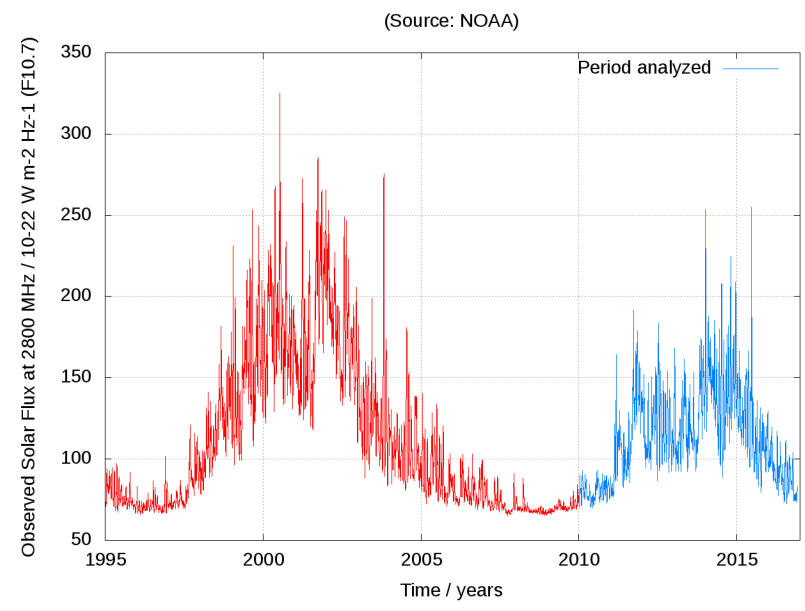

Fig. 5 Evolution of Solar Flux during the period analyzed in this study (blue) compared with the overall evolution since 1995 .

maximum phase of the present Solar Cycle (see Figure 5) and compatible as well with the expected higher ionospheric model error at low latitudes where the equatorial anomaly peaks are located.

The detailed distribution of the UQRG error RMSs and biases can be found in Figures 8 and 9, for VTEC-altimeter and dSTEC-GPS (left- and right-hand plots respectively). It can be seen that the most common error 

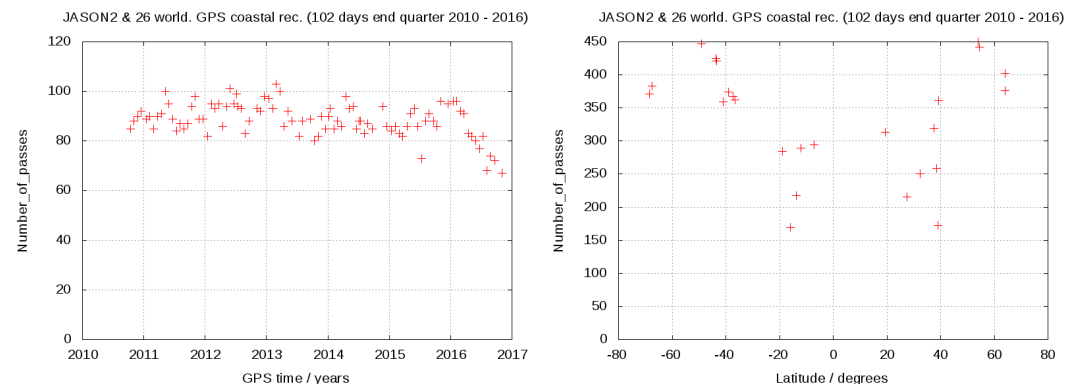

Fig. 6 Number of JASON2 passes processed during this study, versus time (left-hand plot) and versus latitude (right hand plot).
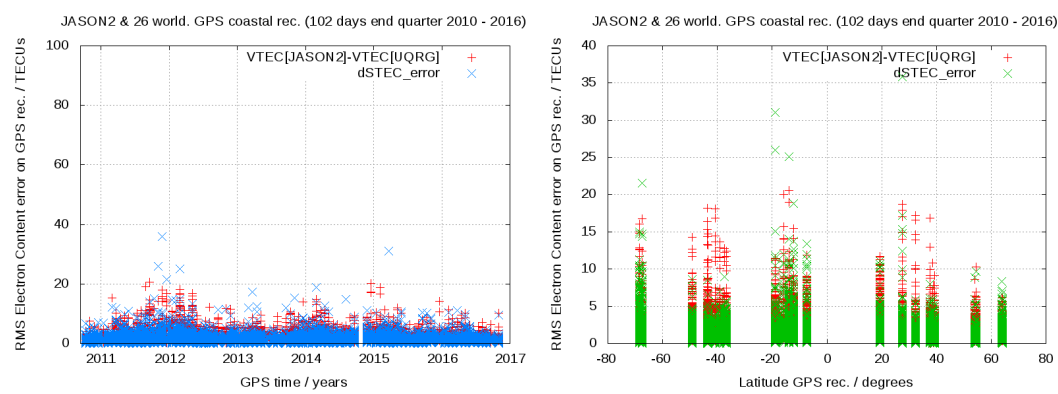

Fig. 7 UQRG GIM model error for both VTEC and dSTEC versus time (left-hand plot) and versus latitude (right-hand plot).
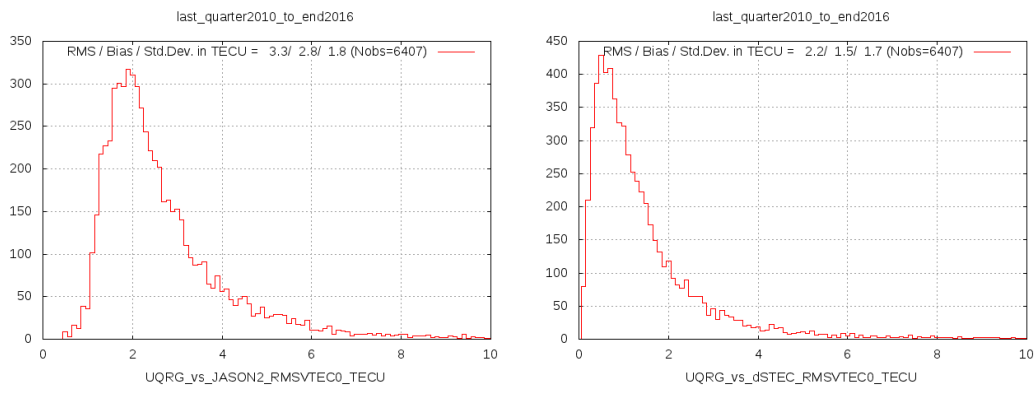

Fig. 8 Histogram of the distribution of UQRG GIM model error RMS values, for each collocated GPS (right hand plot) and JASON2 pass (left hand plot), with a minimum of 25 observations per pass.

RMS values for UQRG and dSTEC are 2 and 0.5 TECUs, respectively. For the biases, the most frequent values are zero or almost zero for dSTEC-GPS, and slightly negative (about -0.5 TECU) for VTEC-altimeter, what is compatible with the well known few TECUs positive bias of altimeters VTEC calibration, combined with the plasmaspheric electron content above the altimeter (see [Azpilicueta and Brunini2009]). 

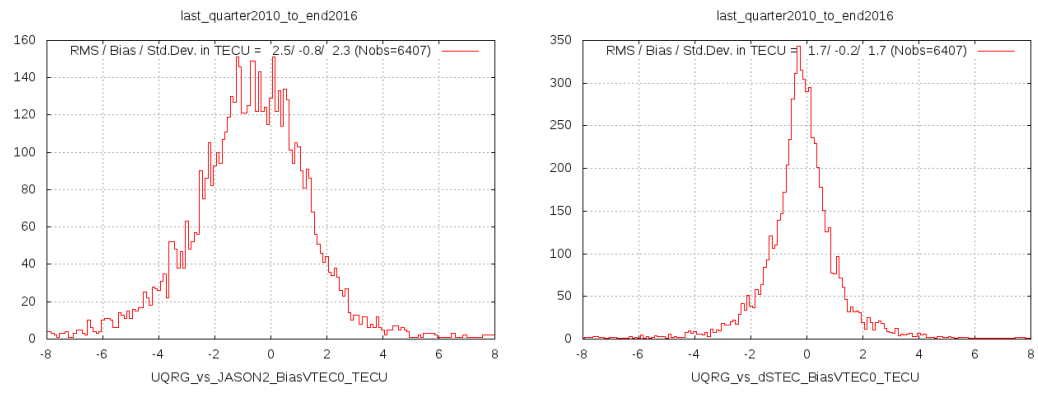

Fig. 9 Histogram of the distribution of UQRG GIM model error bias values, for each collocated GPS (right hand plot) and JASON2 pass (left hand plot), with a minimum of 25 observations per pass.

Table 3 Pearson correlation coefficient between several statistical parameters of dSTECGPS and VTEC-JASON2 errors of UQRG GIM, for different minimum number of measurements per pass over the GPS receivers.

\begin{tabular}{|c|c|c|c|c|c|}
\hline \multicolumn{6}{|c|}{ Pearson correlation coefficient } \\
\hline \# min. & \# passes & Bias & RMS & Std.Dev. & RMSVTECrel \\
\hline 25 & 6407 & 0.16 & 0.36 & 0.29 & 0.19 \\
\hline 50 & 4225 & 0.20 & 0.39 & 0.34 & 0.24 \\
\hline 75 & 2362 & 0.25 & 0.46 & 0.39 & 0.38 \\
\hline 100 & 1180 & 0.27 & 0.44 & 0.35 & $\underline{0.53}$ \\
\hline 150 & 271 & 0.16 & 0.39 & 0.35 & $\underline{0.65}$ \\
\hline 175 & 132 & 0.14 & 0.27 & 0.28 & $\overline{0.63}$ \\
\hline 200 & 69 & 0.18 & $\underline{0.50}$ & $\underline{0.51}$ & 0.78 \\
\hline 225 & 29 & 0.19 & $\overline{0.55}$ & $\overline{0.66}$ & $\underline{0.56}$ \\
\hline
\end{tabular}

In order to characterize the strength of a potential direct one-to-one relationship between both GIM errors, dSTEC-GPS error versus VTEC-altimeter error, the Pearson correlation coefficient is provided in Table 3, for different statistical parameters per pass over one receiver, and for different minimum number of observations per pass. It can be seen that only when exigent conditions on the number of minimum observations per pass are imposed, a relatively high Pearson correlation is obtained, greater than 0.5 for relative RMS error (RMSVTECrel) with $N_{\min } \geq 100$ (reaching up to 0.78 when $N_{\min }=200$ ), and for example for RMS error with $N_{\min } \geq 225$ (you can see in this case the corresponding one-to-one plot in Figure 10).

We can qualitatively justify the tendency to such linear relationship between dSTEC and VTEC relative errors. Indeed, we can approximate the individual dSTEC error $\epsilon[\Delta S]$ as a linear function of the individual VTEC error $\epsilon[V]$, following the development in Equation 2. It is based on the high elevation angle of the reference dSTEC observation (the error of the mapping 


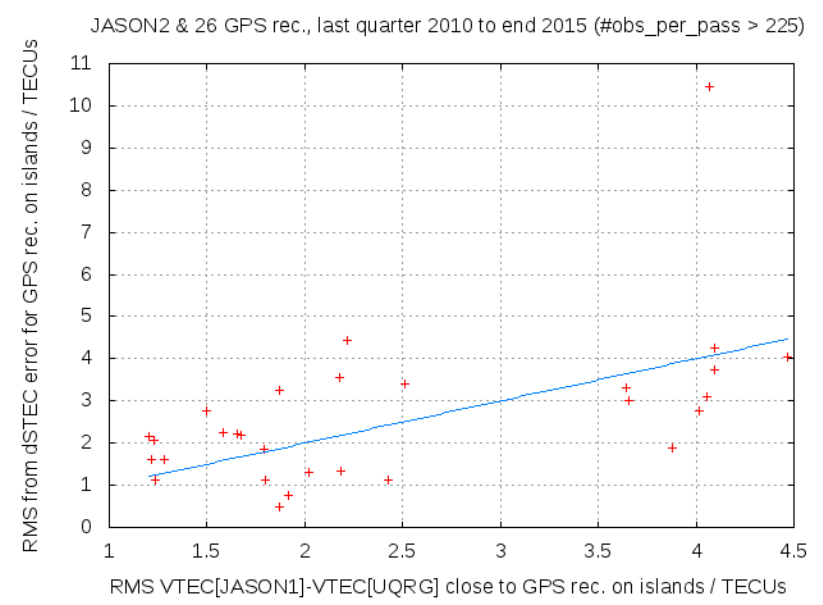

Fig. 10 RMS of UQRG dSTEC discrepancy (referred to observed GPS value) vs RMS of UQRG VTEC discrepancy (vs. JASON2 value) for each one of the collocated passes, and with a minimum number of 225 measurements (the blue line represents the equality of both compared quantities).

function $^{2}, \epsilon[M]$, is then almost zero), assuming VTEC constancy during each (fast and localized) pass of altimeter on the GPS receiver, neglecting as well the lowest elevation ray mapping function error (the toughest hypothesis in spite of $E>15^{\circ}$ ).

$$
\begin{gathered}
\epsilon[\Delta S] \equiv \epsilon\left[S-S\left(E_{\max }\right)\right]= \\
=\epsilon[M \cdot V]-\epsilon\left[M\left(E_{\max }\right) \cdot V\left(E_{\max }\right)\right] \simeq \\
\simeq M \cdot \epsilon[V]+\epsilon[M] \cdot V-M\left(E_{\max }\right) \cdot \epsilon\left[V\left(E_{\max }\right)\right] \approx \\
\approx\left(M-M\left(E_{\max }\right)\right) \cdot \epsilon[V]
\end{gathered}
$$

From Equation 2, and the above mentioned hypothesis, the linear relationship for both error RMS, $R$ is deduced immediately:

$$
R[\epsilon[\Delta S]]=\sqrt{\left\langle\left(M-M\left(E_{\max }\right)\right)^{2}\right\rangle} \cdot R[\epsilon[V]]
$$

where $<>$ represents the average along the collocated altimeter and GPS measurements. Similarly, but in a less approximate way:

$$
\Delta S \approx\left(M-M\left(E_{\max }\right)\right) \cdot V
$$

leading finally to:

$$
R[\Delta S]=\sqrt{\left\langle\left(M-M\left(E_{\max }\right)\right)^{2}\right\rangle} \cdot R[V]
$$

2 Being $M=1 / \sqrt{1-r^{2} \cos ^{2} E / r_{I}^{2}}$ the mapping function, where $r$ and $r_{I}$ are the geocentric distances of the receiver and the ionospheric pierce point (in our case the Earth radius plus $450 \mathrm{~km}$ ) respectively, and $E$ is the elevation angle of the satellite above the receiver spherical horizon (see for instance Equation 32 in [Hernández-Pajares et al.2011]). 
and to a tendency to get similar relative error values between dSTEC-GPS and VTEC-altimeter:

$$
\frac{R[\epsilon[\Delta S]]}{R[\Delta S]} \sim \frac{R[\epsilon[V]]}{R[V]}
$$

\section{Conclusions}

The GPS-based GIMs have been improving in quality since the start of their generation in the nineties of the last century, in spite of the difficulty of providing reliable values at the most part of the ionosphere (such as over the oceans and/or South Hemisphere), far from permanent GPS receivers, and mostly based on the interpolation techniques. In this regard the identification and carefully systematic application of scientifically well-founded techniques of assessment, like the usage of direct and independent dual-frequency altimeter VTEC measurements, and the dSTEC from GPS receiver not taking part in the GIM generation, have played a fundamental role in the evolution of the GIM computation strategies, within a scientific and friendly spirit of cooperation, as has been possible in the International GNSS Service (IGS) since the foundation of its ionospheric working group in 1998.

In this context two independent and complementing ionospheric assessing techniques of VTEC GIMs, taking as reference the direct dSTEC-GPS and VTEC-altimeter observations are, probably for the first time, quantitatively compared.

We have adopted the best performing UPC GIM (UQRG) and we have considered JASON2 altimeter collocated VTEC observations over a set of 26 GPS IGS receivers placed mostly on worldwide islands, during 102 days within almost half solar-cycle, the last quarter of year 2010 up to full year 2016.

The UQRG GIM VTEC and dSTEC errors, derived from collocated JASON2 passes and GPS receivers on islands, present the most frequent RMS values of 2 TECU and 0.5 TECU, repectively. And they tend in general to a certain linear relationship, especially for the error relative error and RMS error when a minimum high number of collocated measurements is present. This result is analytically justified in the manuscript relating to the definitions of both GIM VTEC and dSTEC errors.

Finally it can be confirmed that both complementing and independent assessing techniques, dSTEC-GPS and VTEC-altimeter, successively used in previous works to rank VTEC GIMs, show its quantitative consistency, in coincidence with a simple statistical model, when they are directly compared in collocated scenarios. So the authors strongly recommend its usage (as in a major characterization of ionospheric models we are preparing, involving as well authors from other six analysis centers), when a direct and truly external assessment is required for any electron content model of the ionosphere. 
Acknowledgment

The authors acknowledge the contribution of the four active IGS ionospheric analysis centers, in particular CODE, ESA-ESOC and JPL, for their continuous effort and improvement in their support of the combined IGS GIMs as far as the new analysis centers, NRCAN, CAS and WHU, contributing to IIWG. The English improvements suggested by Mr. Kacper Kotulak are very appreciated as well.

\section{References}

[Azpilicueta and Brunini2009] F. Azpilicueta, C. Brunini, Analysis of the bias between topex and gps vtec determinations. Journal of Geodesy 83(2), 121-127 (2009)

[Bruyninx2004] C. Bruyninx, The euref permanent network: a multi-disciplinary network serving surveyors as well as scientists. GeoInformatics 7(5), 32-35 (2004)

[Colombo et al.2002] O.L. Colombo, M. Hernandez-Pajares, J.M. Juan, J. Sanz, Wide-area, carrier-phase ambiguity resolution using a tomographic model of the ionosphere. Navigation 49(1), 61-69 (2002)

[Dow et al.2009] J.M. Dow, R. Neilan, C. Rizos, The international gnss service in a changing landscape of global navigation satellite systems. Journal of Geodesy 83(3-4), 191-198 (2009)

[Feltens2007] J. Feltens, Development of a new three-dimensional mathematical ionosphere model at european space agency/european space operations centre. Space Weather 5(12) (2007)

[Feltens et al.2011] J. Feltens, M. Angling, N. Jackson-Booth, N. Jakowski, M. Hoque, M. Hernández-Pajares, A. Aragón-Àngel, R. Orús, R. Zandbergen, Comparative testing of four ionospheric models driven with gps measurements. Radio Science 46(6) (2011)

[Feltens2003] J. Feltens, The activities of the ionosphere working group of the international gps service (igs). GPS solutions 7(1), 41-46 (2003)

[Galvan et al.2012] D.A. Galvan, A. Komjathy, M.P. Hickey, P. Stephens, J. Snively, Y. Tony Song, M.D. Butala, A.J. Mannucci, Ionospheric signatures of tohoku-oki tsunami of march 11, 2011: Model comparisons near the epicenter. Radio Science 47(4) (2012)

[Ghoddousi-Fard et al.2011] R. Ghoddousi-Fard, P. Héroux, D. Danskin, D. Boteler, Developing a gps tec mapping service over canada. Space Weather 9(6) (2011)

[Hernández-Pajares et al.2010] M. Hernández-Pajares, et al., Section 9.4 ionospheric model for radio techniques of chapter 9 models for atmospheric propagation delays of iers conventions 2010. IERS Technical Note (2010)

[Hernández-Pajares et al.1999] M. Hernández-Pajares, J. Juan, J. Sanz, New approaches in global ionospheric determination using ground gps data. Journal of Atmospheric and Solar-Terrestrial Physics 61(16), 1237-1247 (1999)

[Hernández-Pajares et al.2009] M. Hernández-Pajares, J. Juan, J. Sanz, R. Orus, A. GarciaRigo, J. Feltens, A. Komjathy, S. Schaer, A. Krankowski, The igs vtec maps: a reliable source of ionospheric information since 1998. Journal of Geodesy 83(3-4), 263-275 (2009)

[Hernández-Pajares et al.2012] M. Hernández-Pajares, J. Juan, J. Sanz, A. Aragón-Àngel, Propagation of medium scale traveling ionospheric disturbances at different latitudes and solar cycle conditions. Radio Science 47(6) (2012)

[Hernández-Pajares2004] M. Hernández-Pajares, IGS Ionosphere WG Status Report: Performance of IGS Ionosphere TEC Maps-Position Paper, in IGS Workshop, Bern, 2004

[Hernández-Pajares et al.2000] M. Hernández-Pajares, J. Juan, J. Sanz, O.L. Colombo, Application of ionospheric tomography to real-time gps carrier-phase ambiguities resolution, at scales of 400-1000 km and with high geomagnetic activity. Geophysical Research Letters 27(13), 2009-2012 (2000) 
[Hernández-Pajares et al.2011] M. Hernández-Pajares, J.M. Juan, J. Sanz, À. AragónÀngel, A. García-Rigo, D. Salazar, M. Escudero, The ionosphere: effects, gps modeling and the benefits for space geodetic techniques. Journal of Geodesy 85(12), 887-907 (2011)

[Hernández-Pajares et al.2014] M. Hernández-Pajares, À. Aragón-Ángel, P. Defraigne, N. Bergeot, R. Prieto-Cerdeira, A. García-Rigo, Distribution and mitigation of higher-order ionospheric effects on precise gnss processing. Journal of Geophysical Research: Solid Earth 119(4), 3823-3837 (2014)

[Hernández-Pajares et al.2016] M. Hernández-Pajares, D. Roma-Dollase, A. Krankowski, R. Ghoddousi-Fard, Y. Yuan, Z. Li, H. Zhang, C. Shi, J. Feltens, A. Komjathy, P. Vergados, S. Schaer, A. Garcia-Rigo, J.M. Gmez-Cama, Comparing performances of seven different global VTEC ionospheric models in the IGS context, in IGS Workshop, Feb. 8-12, 2016, Sydney, Australia, International GNSS Service (IGS), 2016, pp. 1-31. International GNSS Service (IGS)

[Hudnut et al.2001] K.W. Hudnut, Y. Bock, J.E. Galetzka, F.H. Webb, W.H. Young, The southern California integrated GPS network (SCIGN), in The 10th FIG International Symposium on Deformation Measurements, Orange California, USA, 2001, pp. 19-22. Orange California, USA

[Janssen et al.2010] V. Janssen, A. White, T. Yan, CORSnet-NSW: Towards state-wide CORS infrastructure for New South Wales, Australia, in FIG Congress 2010:, 2010, pp. $1-14$

[Juan et al.2012] J. Juan, M. Hernández-Pajares, J. Sanz, P. Ramos-Bosch, R. Orus, W. Ochieng, S. Feng, M. Jofre, P. Coutinho, J. Samson, et al., Enhanced precise point positioning for gnss users. Geoscience and Remote Sensing, IEEE Transactions on 50(10), 4213-4222 (2012)

[Li et al.2015] Z. Li, Y. Yuan, N. Wang, M. Hernandez-Pajares, X. Huo, Shpts: towards a new method for generating precise global ionospheric tec map based on spherical harmonic and generalized trigonometric series functions. Journal of Geodesy 89(4), $331-345(2015)$

[Mannucci et al.1998] A. Mannucci, B. Wilson, D. Yuan, C. Ho, U. Lindqwister, T. Runge, Global mapping technique for gps-derived ionospheric total electron content measurements. Radio science 33(3), 565-582 (1998)

[Orús2005] R. Orús, Contributions on the improvement, assessment and application of the global ionospheric vtec maps computed with gps data. Doctoral dissertation, Ph. D. dissertation. Doctoral Program in Aerospace Science \& Technology (2005)

[Orús et al.2005] R. Orús, M. Hernández-Pajares, J. Juan, J. Sanz, Improvement of global ionospheric vtec maps by using kriging interpolation technique. Journal of Atmospheric and Solar-Terrestrial Physics 67(16), 1598-1609 (2005)

[Rovira-Garcia et al.2015] A. Rovira-Garcia, J.M. Juan, J. Sanz, G. Gonzalez-Casado, A worldwide ionospheric model for fast precise point positioning. Geoscience and Remote Sensing, IEEE Transactions on 53(8), 4596-4604 (2015)

[Sagiya2004] T. Sagiya, A decade of geonet: 1994-2003the continuous gps observation in japan and its impact on earthquake studies. Earth, planets and space 56(8), (2004)

[SCHAER1999] S. SCHAER, Mapping and predicting the earths ionosphere using the Global Positioning System. 1999. 205p, PhD thesis, Ph. D. dissertation. University of Bern, Bern, Switzerland, 1999

[Schaer et al.1996] S. Schaer, G. Beutler, M. Rothacher, T.A. Springer, Daily global ionosphere maps based on GPS carrier phase data routinely produced by the CODE Analysis Center, in Proceedings of the IGS AC Workshop, Silver Spring, MD, USA, 1996, pp. $181-192$

[Snay and Soler2008] R.A. Snay, T. Soler, Continuously operating reference station (cors): history, applications, and future enhancements. Journal of Surveying Engineering 134(4), 95-104 (2008)

[Talaya and Bosch1999] J. Talaya, E. Bosch, CATNET, a permanent GPS network with real-time capabilities, in Proc. ION GPS-99, 12th Int. Tech. Meeting of The Satellite Division of The US Institute of Navigation, 1999, pp. 14-17

[Wells et al.1987] D. Wells, N. Beck, A. Kleusberg, E.J. Krakiwsky, G. Lachapelle, R.B. Langley, K.-p. Schwarz, J.M. Tranquilla, P. Vanicek, D. Delikaraoglou, Guide to GPS positioning, in Canadian GPS Assoc, Citeseer, 1987. Citeseer 
[Zhang et al.2013] H. Zhang, P. Xu, W. Han, M. Ge, C. Shi, Eliminating negative vtec in global ionosphere maps using inequality-constrained least squares. Advances in Space Research 51(6), 988-1000 (2013) 